\title{
EN MEMORIA DEL DR. RICARDO ROSENDO RODRÍGUEZ (1921-2014)
}

\author{
IN MEMORIAM DR. RICARDO ROSENDO RODRÍGUEZ (1921-2014)
}

En julio de 2014 la Sociedad Argentina de Diabetes difundió la noticia del fallecimiento de quien durante muchos años fue para mí el Profesor Rodríguez y desde hace muchos más simplemente Ricardo. Fue una noticia dura, los fallecimientos siempre lo son, pero en este caso tenía el aditamento de haber perdido a alguien por quien sentía gran afecto y una deuda de gratitud.

Con el Dr. Ricardo Rosendo Rodríguez desaparece uno de los últimos discípulos directos del Prof. Bernardo A. Houssay y también uno de sus representantes en las cátedras de Fisiología de las universidades nacionales. Veamos la evidencia de estas afirmaciones.

El Dr. Rodríguez obtuvo su título de médico en la Facultad de Medicina de la Universidad de Buenos Aires (UBA) en 1951. Rápidamente entró en contacto con la Escuela de Fisiología del Prof. Houssay y para completar su formación en el área de investigación básica, en 1955 fue a trabajar (beca Fundación Rockefeller) al Departamento de Química Fisiológica de la Universidad de Yale, New Haven, Conn (Estados Unidos), bajo la dirección del Prof. Dr. CNH Long. En ese momento el Dr. Long era una figura de enorme prestigio en el área de la fisiología del músculo.

A su regreso completó su trabajo de tesis doctoral sobre la "Acción de las glándulas y hormonas sexuales en el metabolismo de los hidratos de carbono y en la diabetes", bajo la dirección del Prof. Dr. Bernardo Houssay. El trabajo obtuvo la calificación "sobresaliente"; recibió el Premio Facultad de Medicina lo que le permitió acceder al título de Doctor en Medicina de la UBA.

Al año siguiente de doctorarse, fue designado Prof. Titular de Fisiología y Director del Instituto de Fisiología de la Facultad de Ciencias Médicas de la Universidad Nacional de La Plata (UNLP), cargo que desempeñó hasta 1970. En dicho Instituto, y junto con el Dr. Rodolfo R. Brenner, pusieron la simiente de la investigación básica en dicha Facultad: se crearon cargos de dedicación exclusiva, se obtuvieron subsidios nacionales y extranjeros para financiar proyectos de investigación básica y se estableció un sistema de reclutamiento de alumnos destacados para conformar los planteles docentes de Fisiología y Bioquímica. Estas acciones crearon paulatina- mente un ambiente que atrajo a investigadores ya formados en diferentes disciplinas (médicas y no médicas) y a jóvenes graduados que "descubrieron" a la investigación científica como una meta para su labor futura. El resultado ha sido que se generaron condiciones que favorecieron el desarrollo de tesis doctorales con temas de investigación originales y la conformación de un plantel docente en materias básicas que comparte docencia con investigación bajo el régimen de dedicación exclusiva, hecho inédito en aquellos tiempos en esa Facultad.

Estas actividades se complementaron con el envío de jóvenes doctores a centros de investigación de excelencia en Estados Unidos y Europa, que a su regreso desarrollaron nuevas líneas de investigación que ampliaron y complementaron la tradicional línea de diabetes. En ese contexto surgieron nombres como Horacio Cingolani, Sergio Blessa, Héctor Buschiazzo, Norberto González, Perla Mordujovich, Nelva Tacconi y posteriormente Ricardo Calandra, Ricardo Coccozella, Alberto Musante, María J. Tacconi y quien redacta este artículo, entre otros. En su mayoría todos siguieron trabajando en investigación y creando grupos de buena producción científica en el país o en Estados Unidos.

En 1961 el Dr. Rodríguez ingresó a la Carrera de Investigador del CONICET de reciente creación y como miembro presentó numerosos trabajos científicos originales que fueron publicados en revistas especializadas nacionales y extranjeras al igual que varios libros relacionados con las ciencias fisiológicas y la diabetes experimental. De igual modo, dirigió 14 tesis doctorales.

En el período 1968-1970 fue elegido Decano de la Facultad de Ciencias Médicas de la UNLP, cargo desde el cual continúo su apoyo a la investigación básica y la formación de recursos humanos. También participó activamente en la finalización del edificio actual de esa Facultad. No logró, en cambio, la simbiosis entre las ciencias básicas y clínicas manteniéndose la tradicional falta de diálogo entre ambas disciplinas que aún hoy, aunque en forma más atenuada, sigue vigente.

En 1971 dejó la ciudad de La Plata al ser nombrado Prof. Titular y Director del Instituto de Fisiología Prof. Dr. Bernardo A. Houssay de la Facultad de 
Medicina de la UBA, cargo en el que permaneció hasta 1989. Ese lapso coincidió con un período de alternancias, turbulencias y divisiones políticas muy intensas al cual no fue ajena la Universidad, hechos en los que se vio involucrado y determinaron su alejamiento de la institución en forma conflictiva.

También en ese período actuó como Subsecretario de Coordinación Universitaria en el Ministerio de Cultura y Educación de la Nación bajo la conducción del Dr. Gustavo Malek. Gracias a su gestión, se fundaron varias universidades nacionales como la de Comahue, Luján, NOA y Santa Rosa. Dichas universidades se crearon después de un estudio exhaustivo (factibilidad técnica, cantidad de habitantes, posibilidad de desarrollo) y permitieron que miles de estudiantes no debieran emigrar a otras provincias para lograr una formación universitaria.

En 1977 fue designado Prof. Titular de Fisiología y Biofísica de la Facultad de Medicina de la Universidad del Salvador, cargo en el que permaneció hasta su fallecimiento. En el período 20012008 amplió su labor docente a nivel de postgrado desempeñándose como creador y Director de la Maestría en Diabetes en esa misma Facultad.

Durante su extensa actividad académica y científica recibió numerosos premios y distinciones tales como Miembro Correspondiente de la Academia Nacional de Ciencias de Buenos Aires, Argentina (1972); Caballero de la Orden del Corpus Christi, Toledo, España (1973); Profesor Visitante de la Universidad de Japón (1978); Miembro de la Academia de Medicina de Córdoba, Argentina (1984); Profesor Honorario de la Universidad del Uruguay (1984); Profesor Visitante de la Universidad de UML (1989); Maestro de la Medicina, especialidad en Fisiología y Diabetes Experimental de la Sociedad Médica de La Plata, Argentina (2003); Doctor Honoris Causa de la Facultad de Medicina y Farmacia de la Universidad de Cracovia, Rumania (2003); Maestro de la Medicina de la Universidad del Salvador, Argentina (2005); y Maestro de las Ciencias, las Artes y la Investigación en Medicina otorgado por la Sociedad Argentina de Farmacología y Terapéutica de la Asociación Médica Argentina (2007).

Su actividad a nivel de sociedades científicas fue también intensa: se desempeñó como presidente de la Sociedad Argentina de Diabetes (1965-1966), de la Fundación Bernardo Houssay (1992-2002) y de la Asociación Argentina de Fisiopatología (20022006), además de presidente Honorario de la Sociedad Argentina de Farmacología y Terapéutica de la Asociación Médica Argentina (2001-2014), miembro del Consejo de la Unión Internacional de Ciencias Fisiológicas (IUPS)(1980-1989), del Consejo de la Sociedad Internacional de Fisiopatología (ISP) (2002-2010) y del Tribunal de Honor de la Sociedad Argentina de Farmacología y Terapéutica de la Asociación Médica Argentina (2002-2014).

Esta simple enumeración cronológica de los logros académicos y científicos alcanzados por el Dr. Rodríguez demuestra que su paso por este mundo no fue desapercibido: su impronta mayor no esté quizás dada por sus trabajos y cargos desempeñados, sino por la enorme contribución a la promoción de un sistema de investigación de medicina básica en la Facultad de Ciencias Médicas de la UNLP, que aún sigue vigente y se multiplica a través de quienes él inició en este ámbito. Varios de sus discípulos continuaron su labor fuera del país por lo que su tarea trascendió el ámbito local. Todo ello demuestra que si hay algo que de él no puede decirse es que fue "tibio" y en consecuencia su accionar creó adeptos y detractores, amigos y adversarios, pero su obra existe y es verificable.

Sin embargo, ¿es esta obra debidamente reconocida por sus pares? Creo que no, y son varias las razones de su desconocimiento. Entre ellas que su máximo éxito lo tuvo hace casi 50 años y nuestra memoria es frágil, y que los hechos ocurrieron fuera del gran escenario nacional que es Buenos Aires. Igualmente porque le tocó vivir épocas de importante turbulencia política y tomó parte activa en ellas por lo cual lastimó y fue lastimado dejando de ambos lados heridas difíciles de restañar. ¿Actitudes reprochables? Quizás, ¿pero quién puede en este ámbito arrojar la primera piedra?

Una mirada retrospectiva y un análisis desapasionado de la vida del Dr. Rodríguez permitiría tomar como ejemplo a imitar sus aspectos más positivos. También reconocer que las diferencias suelen ser complementarias y la integración de diversos puntos de vista optimiza funciones. La fisiología, disciplina que cultivó, lo demuestra continuamente: por ejemplo, la visión del ojo derecho es diferente a la del ojo izquierdo, pero su integración a nivel cortical logra la visión estereoscópica. Ojalá los argentinos, por lo menos a nivel de nuestra familia diabetológica, aprendamos a compartir y convivir en un ámbito de respeto mutuo y creatividad. Esto hará que la obra de personas como la del Dr. Rodríguez se magnifique y contribuya a tener una sociedad más justa y saludable.

Juan José Gagliardino 